\title{
Animal Model of Lung Metastasis of Hepatocellular Carcinoma: A Tool for the Development of Anti-Metastatic Therapeutics*
}

\author{
Mitsuru Futakuchi \\ Department of Molecular Toxicology, Graduate School of Medical Sciences, Nagoya City University, Nagoya, Japan. \\ Email: futakuch@med.nagoya-cu.ac.jp
}

Received December $19^{\text {th }}, 2012$; revised January $18^{\text {th }}, 2013$; accepted January $27^{\text {th }}, 2013$

\begin{abstract}
We observed that N-nitrosomorpholine (NMOR) given after a multi-carcinogenic treatment induced liver carcinomas with 56\% lung metastasis. An additional treatment with diethylnitrosamine (DEN) with NMOR further enhanced the incidence of hepatocellular carcinoma (HCC) with lung metastasis. We have further revised the duration of NMOR treatment to establish an animal model with a simple experimental protocol and an appropriate experimental duration to facilitate investigation exploring the mechanisms of HCC metastasis and development of anti-metastatic therapeutics. We observed that DEN exposure followed by a 16-week treatment with NMOR to be a most efficient protocol for the induction of HCC metastasizing to the lung. In this review, we will discuss about the usefulness of animal models for induction of highly metastatic HCC and the assessment of the efficacy of anti-metastatic therapeutics. Additionally, we will also discuss use of these models in analysis of individual steps in the metastatic process by using non-steroidal anti-inflammatory drugs, aspirin and indomethacin, two nuclear factor kappa B (NF- $\kappa \mathrm{B}$ ) inhibitors, pentoxifylline and $\mathrm{N}$-acetyl-L-cysteine.
\end{abstract}

Keywords: Lung Metastasis; Hepatocellular Carcinoma; NF- $\kappa$ B Inhibitor

\section{Introduction}

Despite the continuous improvements in early diagnosis and therapy for early stage cancer, most deaths from cancer occur due to metastases [1]. Once metastatic disease has developed, aggressive treatment such as systemic chemotherapy is required since surgical removal of all metastatic foci is not feasible [2]. Therefore, it is necessary to identify and develop novel treatment strategies for preventing cancer metastasis.

Tumor metastasis is a multistage process during which malignant cells spread from the primary tumor to discontiguous organs [3]. It involves invasion, transport, arrest, adherence, extravasation, growth in different microenvironments, which are treated clinically with different strategies depending on the tumor histiotype and metastatic location [4].

To study the mechanisms underlying metastasis, many tools and models have been developed. Most of them use cancer cell lines or transplantable tumors, injected into blood vessels or intraperitoneal cavity, or transplanted into the cecum, spleen or subcutis [5-7]. These models have provided very useful tools for analysis of individual

\footnotetext{
*The authors have declared that no conflict of interest exists.
}

steps in the metastatic process. However, in order to assess the efficacy of therapeutic treatments for advanced cancers with metastasis, it is necessary to develop animal cancer models for natural course of metastasis, which feature frequent metastasis of primary tumors to distant organs. Thus, comprehensive analysis is required to develop anti-metastasis agents.

\section{Establishment of an in Vivo Highly Metastatic Rat HCC Model}

We have previously shown by chance that N-nitrosomorpholine (NMOR) given after a multi-carcinogenic treatment with N-diethylnitrosamine (DEN), N-methylnitrosourea (MNU), N-butyl-N-(4-hydroxybutyl)nitrosamine (BBN), 1, 2-dimethyl-hydrazine (DMH), and 2, 2'-dihydroxy-di-N-propylnitrosaminee (DHPN) induces liver carcinomas with frequent lung metastasis [8]. We attempted to establish an animal model with a simple experimental protocol and an appropriate experimental duration which would facilitate further study of the mechanisms of metastasis and antimetastatic agents (Figure 1) [9].

NMOR and DEN have been widely used as hepatocarcinogens in animal models, and the induced malignant 


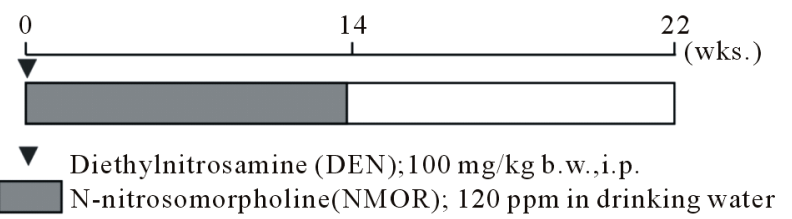

Figure 1. Protocol for an in vivo highly metastatic rat HCC model We had established an in vivo lung metastasis model of HCC induced by two hepato-carcinogens, DEN and 120 ppm NMOR. This model allows us to apply chemical substance in the intervening period to investigate modifying factors, particularly those leading to inhibition of lung metastasis formation. We attempted to establish an animal model with a simple experimental protocol and an appropriate experimental duration which would facilitate further study of the mechanisms of metastasis and antimetastatic agents.

tumors have been well characterized [10-12]. Lung metastasis by induced HCC in rats given either DEN or NMOR has been reported by Lijinsky et al. $[13,14]$. In our previous study, treatment with NMOR alone or with DEN followed by 8-weeks NMOR resulted HCC induction (Figure 2(a)) with only few lung metastases (Figure 2(b)) [9]. In contrast, DEN followed by 16 or 22-weeks NMOR treatment was associated HCC (Figure 2(c)) with higher frequencies of lung metastases (Figure 2(d)), with a duration dependence of NMOR treatment [9]. Histologically, we observed not only large metastatic nodules, but also extravasation in the lung at week 22 . These findings suggest that a multi step process of metastasis (including invasion, transport, arrest, adherence, extravasation, and tumor cell proliferation) proceeded between weeks 16 and 22. Therefore, using this model, chemical substances could be applied in the intervening period to investigate modifying factors, particularly those leading to inhibition of lung metastasis formation.

Change in the expression of cadherin, a major adhesion molecule of epithelia [15-17], has been implicated in carcinogenesis because loss is frequent in human and murine high grade epithelial cancers [18-20]. In the previous study, we found that pan-cadherin expression to be decreased in the order of adenoma, HCC and advanced HCC. The quantitative difference of cadherin expression was observed between the HCC with metastasis and that without metastasis. These results suggest that downregulation of cadherin expression may occur as an early event of carcinogenesis with decrease with in line with hyperplasia, adenoma and HCC.

Detection of circulating tumor cells in the blood may give us the evidence that tumor cells had already entered in the circulation before microscopic metastasis lesions were detected, and circulating tumor cells were also assessed in relation to HCC development and lung metastasis formation [21]. For detection of circulating

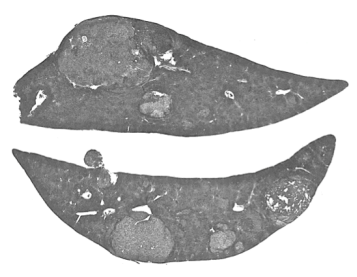

(a)

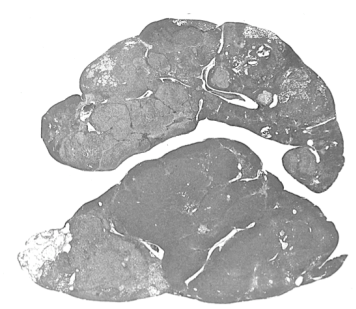

(c)

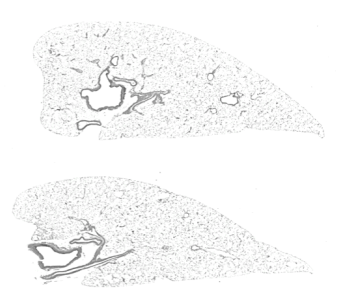

(b)

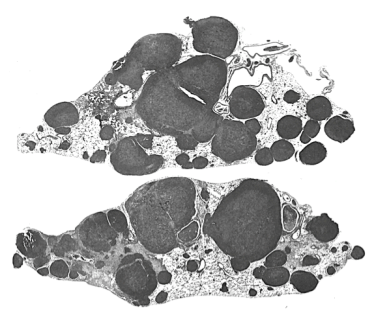

(d)
Figure 2. HCC and lung metastasis formation (a) Treatment with NMOR alone or with DEN followed by 8-weeks NMOR resulted HCC induction in week14; (b) In week 14, we observed only few lung metastases; (c) DEN followed by 14-weeks NMOR treatment induced multiple HCC; (d) We observed not only large metastatic nodules, but also extravasation in the lung at week 22.

tumor cells, RT-PCR has been utilized [22-24], and we found CK-8 expression have been demonstrated to be positive in blood. Through the travel in the circulation, only a small percentage of tumor cells $(<0.01 \%)$ released from a primary tumor survive and arrest in the capillary beds of distant organs producing a successful metastasis [25]. Survival in the circulation appears to be responsible for this inefficiency due to immune factors in the blood, and this response may be the reason why tumor cells are circulating in the blood while no microscopic metastasis was found.

\section{Suppression of Lung Metastasis by Aspirin but Not Indomethacin in an in Vivo Model of Chemically-Induced HCC}

Because the metastatic cascade is a continuous process which begins with proliferation of the primary tumor and ends with proliferation of the metastatic foci [26], we hypothesized interference with cell proliferation might prevent metastasis formation. Nonsteroidal anti-inflammatory drugs (NSAIDs) such as aspirin (ASP) and indomethacin (IM) are well known as potential chemopreventive agents through their modulation of levels of prostaglandins, PGE2, and cyclooxygenase (COX) in the colon and also other organs $[27,28]$.

We have demonstrated that ASP but not IM significantly reduced the severity of lung metastasis, but not the average number. This indicates that the effect of ASP 
was marginal [29]. We also demonstrated that only ASP suppressed lung metastasis formation although ASP and IM exerted inhibitory effects on cell proliferation of HCCs [29]. Thus, it is suggested that inhibition of cell proliferation per se may not be involved in the mechanism of inhibition of lung metastasis by ASP.

Epidemiological studies revealed that NSAIDs, such as ASP and IM, which suppress COX activity, possess considerable potential as chemopreventive agents for colorectal cancer [30,31]. Constitutive expression of COX-2 has been demonstrated to lead to phenotypic changes that alter the metastatic potential of colorectal cancer cells [32], and COX-2 inhibitor was found to exert inhibitory effects on metastasis formation of various cancer $[33,34]$. However, our data demonstrated that IM did not suppress lung metastasis formation in spite of down-regulation of COX-2 [29], indicating no direct involvement of this enzyme in the inhibitory effect on HCC metastasis. In addition, neither ASP nor IM exerted any apparent influence on cadherin expression within HCC [29]. Therefore, the mechanism of inhibition by ASP might be mainly in a stage of the metastatic cascade after the primary site, such as attachment to the vascular endothelium or re-invasion or re-proliferation in the lung.

The attachment of a cancer cell to the vascular endothelium is a complex phenomenon involving a number of cell adhesion molecules (CAMs). Among these latter, E-selectin, ICAM-1 and VCAM-1 are considered to play primary roles in hematogenous metastasis $[35,36]$. Induction of Eselectin, ICAM-1 and VCAM-1 is mediated by the transcription factor nuclear factor-kappa B (NF- $\kappa$ B) $[37,38]$. ASP has been shown to inhibit NF- $\kappa$ B dependent transcription [39], and these transcriptions appear not to be related to the inhibition of COX activity, since IM was ineffective [40]. In the previous study, ASP significantly suppressed the expressions of ICAM-1 and VCAM-1 [29], indicating a probable role of inhibition of attachment of tumor cells to the vascular endothelium. Therefore, a stronger inhibitor of NF- $\kappa$ B might be expected to have a stronger inhibitory effect on lung metastasis formation.

\section{Suppression of Metastasis by Nuclear Factor KappaB Inhibitors in an in Vivo Lung Metastasis Model of HCC}

In order to evaluate the suppressive effects of NF- $\kappa \mathrm{B}$ inhibitors, we examined three examples, pentoxifylline (PTX) [41], Nacetyl-L-cysteine (NAC) [42], and ASP [39], in our in vivo lung metastasis model. PTX, widely used as a hemorheological agent in the treatment of peripheral vascular disease, was earlier shown to suppress lung metastasis formation by B16F10 melanoma [43] and NAC inhibits VEGF production in human melanoma cell lines [44], invasion of endothelial cells [45], and invasion of human bladder cancer cells through the suppression of MMP-9 [46]. ASP has been demonstrated to inhibit angiogenesis [47] and HGF-induced invasiveness of HepG2 human hepatoma cells [48].

Among the NF- $\kappa \mathrm{B}$ inhibitors, PTX exerted the strongest effects on lung metastasis formation and NAC had rather less influence, while ASP did not significantly reduce lung metastasis [49]. Although PTX and NAC suppressed lung metastasis, they did not improve the survival rates. This was mainly because the increase in the mortality rates owing to bleeding from primary HCC diminished the decrease that resulted from suppression of lung metastasis. Thus, the increase and decrease were not significant, and treatment with NF- $\kappa$ B inhibitors did not affect the incidences and multiplicities of HCCs in liver. Therefore, further studies are necessary to elucidate the reasons why PTX and NAC did not affect the survival rates.

To evaluate the degree of inhibition of NF- $\kappa \mathrm{B}$ transcription, inhibitor of $\kappa \mathrm{B}(\mathrm{I} \kappa \mathrm{B})$ protein levels in HCCs were evaluated by western blotting. The $\mathrm{I} \kappa \mathrm{B}$ family has been shown to control the function of NF- $\kappa \mathrm{B}$ complexes [50,51], and IkB protein has been shown to activate NF$\kappa \mathrm{B}$ when it is phosphorylated or cleaved by proteasomes through a ubiquitine- dependent pathway [52,53]. We demonstrated that $\mathrm{I} \kappa \mathrm{B}$ protein expression was suppressed by test compounds in the order of PTX, NAC and ASP. Therefore, these results suggest that the mechanism of reduction of lung metastasis formation observed in this study may involve inhibition of NF- $\kappa$ B transcription.

The contribution of NF- $\kappa$ B to the process of metastasis has been explored in relation to CAMs and VEGF expression was found to be significantly suppressed by NF- $\kappa$ B signaling blockade [54], and promoted by coactivation of NF- $\kappa$ B [55]. PTX significantly suppressed expression of VEGF-A splicing variants with heparin-, heparin-sulfate-, and extracellular matrix-binding domains. These results suggest that the mechanism of the suppression of lung metastasis by PTX involves suppression of VEGF-A with heparin-binding domains. On the other hand, NAC, which had less influence on lung metastasis formation than PTX, suppressed VEGF-A variants with and without the heparin-binding domain. Therefore, whether NF$\kappa \mathrm{B}$ controls only VEGF-A with heparin-binding domains remains to be elucidated.

\section{Conclusions}

Our rat model presented here provides an excellent tool for rapid induction of metastatic HCC. To our knowledge, this is the first model to reflect the natural course 




Figure 3. A multi step process of metastasis and therapeutic targets of NF- $\boldsymbol{k}$ B inhibitors A multi step process of metastasis (including invasion, transport, arrest, adherence, extravasation, and tumor cell proliferation) proceeded between weeks 16 and 22. Therefore, using this model, chemical substances could be applied in the intervening period to investigate modifying factors, particularly those leading to inhibition of lung metastasis formation. NF- $\kappa$ B inhibitors have the potential to inhibit lung metastasis of rat HCCs in vivo, with PTX being the most promising candidate. They may interfere with attachment of tumor cells to the vascular endothelium at metastatic sites, and decrease of VEGFA188 may play an important role.

of malignant tumors which metastasize to lung, and this model should be applicable not only for the elucidation of mechanisms underlying metastasis, but also to test anti-metastatic agents.

ASP, but not IM, has the potential to inhibit lung metastasis by rat HCC in vivo, the mechanism apparently involving neither inhibition of cell proliferation nor detachment from primary tumors. Inhibition of attachment to the vascular endothelium in the lung is more likely to be the mechanism responsible for the suppression of lung metastasis formation by ASP. NF- $\kappa \mathrm{B}$ inhibitors have the potential to inhibit lung metastasis of rat HCCs in vivo, with PTX being the most promising candidate. They may interfere with attachment of tumor cells to the vascular endothelium at metastatic sites, and decrease of VEGFA188 may play an important role (Figure 3).

This in vivo model for induction of rat highly metastatic hepatocellular carcinomas is clearly a useful tool for the assessment of the efficacy of therapeutic treatments for metastasis formation and for analysis of individual steps in the metastatic process.

\section{Acknowledgements}

The author thanks Dr. Rakesh K. Singh (Department of Pathology and Microbiology, University of Nebraska Medical Center) for expert suggestions and comments. This work was supported in part by a research grant from Scientific Support Programs for Cancer Research Grantin-Aid for Scientific Research on Innovative Areas Ministry of Education, Culture, Sports, Science and Tech- nology, and a grant-in-aid for Scientific Research (C) from the Japan Society for Promotion of Science.

\section{REFERENCES}

[1] A. Jemal, F. Bray, M. M. Center, J. Ferlay, E. Ward and D. Forman, "Global Cancer Statistics," CA: A Cancer Journal for Clinicians, Vol. 61, No. 2, 2011, pp. 69-90. doi:caac.20107[pii]10.3322/caac.20107

[2] D. B. Walsh, S. Downing, R. Nauta and M. N. Gomes, "Metastatic Cancer. A Relative Contraindication to Vena cava Filter Placement,” Cancer, Vol. 59, No. 1, 1987, pp. 161-163.

doi:10.1002/1097-0142(19870101)59:1<161::AID-CNCR 2820590131>3.0.CO;2-A

[3] I. J. Fidler, "Critical Factors in the Biology of Human Cancer Metastasis: Twenty-Eighth G.H.A. Clowes Memorial Award Lecture,” Cancer Research, Vol. 50, No. 19, 1990, pp. 6130-6138.

[4] J. E. Talmadge and I. J. Fidler, “AACR Centennial Series: The Biology of Cancer Metastasis: Historical Perspective,” Cancer Research, Vol. 70, No. 14, 2010, pp. 56495669. doi:10.1158/0008-5472.CAN-10-1040

[5] K. Morikawa, S. M. Walker, J. M. Jessup and I. J. Fidler, "In Vivo Selection of Highly Metastatic Cells from Surgical Specimens of Different Primary Human Colon Carcinomas Implanted into Nude Mice,” Cancer Research, Vol. 48, 1988, pp. 1943-1948.

[6] X. B. Li, J. Hamada, N. Takeichi, M. Hosokawa and H. Kobayashi, "Timing of UFT Administration Combined with Surgical Operation in Pulmonary Metastasis of Rat Mammary Carcinoma,” Japanese Journal of Cancer and Chemotherapy, Vol. 16, No. 7, 1989, pp. 2381-2385.

[7] I. J. Fidler and M. L. Kripke, "Metastasis Results from Preexisting Variant Cells within a Malignant Tumor," Science, Vol. 197, No. 4306, 1977, pp. 893-895. doi:10.1126/science.887927

[8] T. Masui, H. Nakanishi, K. Inada, T. Imai, Y. Mizoguchi, H. Yada, M. Futakuchi, T. Shirai and M. Tatematsu, "Highly Metastatic Hepatocellular Carcinomas Induced in Male F344 Rats Treated with N-Nitrosomorpholine in Combination with Other Hepatocarcinogens Show a High Incidence of p53 Gene Mutations Along with Altered mRNA Expression of Tumor-Related Genes," Cancer Letters, Vol. 112, No. 1, 1997, pp. 33-45. doi:10.1016/S0304-3835(96)04543-0

[9] M. Futakuchi, M. Hirose, T. Ogiso, K. Kato, M. Sano, K. Ogawa and T. Shirai, "Establishment of an in Vivo Highly Metastatic Rat Hepatocellular Carcinoma Model,” Japanese Journal of Cancer and Chemotherapy, Vol. 90, No. 11, 1999, pp. 1196-1202. doi:10.1111/j.1349-7006.1999.tb00695.x

[10] H. Enzmann, H. Zerban, S. A. Kopp, E. Loser and P. Bannach, "Effects of Low Doses of N-Nitrosomorpholine on the Development of Early Stages of Hepatocarcinogenesis," Carcinogenesis, Vol. 16, No. 7, 1995, pp. 15131518. doi:10.1093/carcin/16.7.1513

[11] M. Volm, H. Zerban, J. Mattern and T. Efferth, "Overexpression of P-Glycoprotein in Rat Hepatocellular Carci- 
nomas Induced with N-Nitrosomorpholine," Carcinogenesis, Vol. 11, No. 1, 1990, pp. 169-172. doi:10.1093/carcin/11.1.169

[12] E. Weber and P. Bannasch, "Dose and Time Dependence of the Cellular Phenotype in Rat Hepatic Preneoplasia and Neoplasia Induced by Continuous Oral Exposure to NNitrosomorpholine," Carcinogenesis, Vol. 15, No. 6, 1994, pp. 1235-1242. doi:10.1093/carcin/15.6.1235

[13] W. Lijinsky, "Metastasizing Tumors in Rats Treated with Alkylating Carcinogens," Carcinogenesis, Vol. 16, No. 4, 1995, pp. 675-681. doi:10.1093/carcin/16.4.675

[14] W. Lijinsky, R. M. Kovatch, C. W. Riggs and P. T. Walters, "Dose-Response Study with N-Nitrosomorpholine in Drinking Water of F-344 Rats,” Cancer Research, Vol. 48, No. 8, 1988, pp. 2089-2095.

[15] M. Takeichi, "Cadherin Cell Adhesion Receptors as a Morphogenetic Regulator,” Science, Vol. 251, No. 5000, 1991, pp. 1451-1455. doi:10.1126/science.2006419

[16] M. Takeichi, M. Watabe, S. Shibamoto and F. Ito, "Cadherin-Dependent Organization and Disorganization of Epithelial Architecture," Princess Takamatsu Symposia, Vol. 24, 1994, pp. 28-37.

[17] M. Takeichi, "Morphogenetic Roles of Classic Cadherins," Current Opinion in Cell Biology, Vol. 7, No. 5, 1995, pp. 619-627. doi:10.1016/0955-0674(95)80102-2

[18] R. Umbas, J. A. Schalken, T. W. Aalders, B. S. Carter, H. F. Karthaus, H. E. Schaafsma, F. M. Debruyne and W. B. Isaacs, "Expression of the Cellular Adhesion Molecule E-Cadherin Is Reduced or Absent in High-Grade Prostate Cancer," Cancer Research, Vol. 52, No. 18, 1992, pp. 5104-5109.

[19] P. Navarro, M. Gomez, A. Pizarro, C. Gamallo, M. Quintanilla and A. Cano, "A Role for the E-Cadherin cell-Cell Adhesion Molecule during Tumor Progression of Mouse Epidermal Carcinogenesis," The Journal of Cell Biology, Vol. 115, No. 2, 1991, pp. 517-533. doi:10.1083/jcb.115.2.517

[20] S. Wakatsuki, R. Watanabe, K. Saito, T. Saito, A. Katagiri, S. Sato and Y. Tomita, "Loss of Human E-Cadherin (ECD) Correlated with Invasiveness of Transitional Cell Cancer in the Renal Pelvis, Ureter and Urinary Bladder,” Cancer Letters, Vol. 103, No. 1, 1996, pp. 11-17. doi:10.1016/0304-3835(96)04194-8

[21] H. Yoshino, M. Futakuchi, Y. M. Cho, K. Ogawa, F. Takeshita, N. Imai, S. Tamano and T. Shirai, "Modification of an in Vivo Lung Metastasis Model of Hepatocellular Carcinoma by Low Dose N-Nitrosomorpholine and Diethylnitrosamine," Clinical and Experimental Metastasis, Vol. 22, No. 5, 2005, pp. 441-447. doi:10.1007/s10585-005-2807-9

[22] S. Ito, H. Nakanishi, T. Hirai, T. Kato, Y. Kodera, Z. Feng, Y. Kasai, K. Ito, S. Akiyama, A. Nakao and M. Tatematsu, "Quantitative Detection of CEA Expressing Free Tumor Cells in the Peripheral Blood of Colorectal Cancer Patients during Surgery with Real-Time RT-PCR on a LightCycler," Cancer Letters, Vol. 183, No. 2, 2002, pp. 195-203. doi:10.1016/S0304-3835(02)00157-X

[23] M. Matsumura, Y. Niwa, Y. Hikiba, K. Okano, N. Kato,
S. Shiina, Y. Shiratori and M. Omata, "Sensitive Assay for Detection of Hepatocellular Carcinoma Associated Gene Transcription (Alpha-Fetoprotein mRNA) in Blood," Biochemical and Biophysical Research Communications, Vol. 207, No. 2, 1995, pp. 813-818. doi:10.1006/bbrc.1995.1259

[24] Y. Miyajima, K. Horibe, M. Fukuda, K. Matsumoto, S. Numata, H. Mori and K. Kato, "Sequential Detection of Tumor Cells in the Peripheral Blood and Bone Marrow of Patients with Stage IV Neuroblastoma by the Reverse Transcription-Polymerase Chain Reaction for Tyrosine Hydroxylase mRNA,” Cancer, Vol. 77, No. 6, 1996, pp. 1214-1219.

doi:10.1002/(SICI)1097-0142(19960315)77:6<1214::AID -CNCR31>3.0.CO;2-2

[25] K. J. Luzzi, I. C. MacDonald, E. E. Schmidt, N. Kerkvliet, V. L. Morris, A. F. Chambers and A. C. Groom, "Multistep Nature of Metastatic Inefficiency: Dormancy of Solitary Cells after Successful Extravasation and Limited Survival of Early Micrometastases," American Journal of Pathology, Vol. 153, No. 3, 1998, pp. 865-873. doi:10.1016/S0002-9440(10)65628-3

[26] I. J. Fidler, "The Pathogenesis of Cancer Metastasis: The 'Seed and Soil' Hypothesis Revisited," Nature Reviews Cancer, Vol. 3, No. 6, 2003, pp. 453-458. doi:10.1038/nrc1098

[27] A. T. Chan and N. R. Cook, "Are We Ready to Recommend Aspirin for Cancer Prevention?” Lancet, Vol. 379, No. 9826, 2012, pp. 1569-1571. doi:10.1016/S0140-6736(11)61654-1

[28] T. F. Imperiale, "Aspirin and the Prevention of Colorectal Cancer,” The New England Journal of Medicine, Vol. 348, 2003, pp. 879-880. doi:10.1056/NEJMp030005

[29] M. Futakuchi, K. Ogawa, M. Sano, S. Tamano, F. Takeshita and T. Shirai, "Suppression of Lung Metastasis by Aspirin but Not Indomethacin in an in Vivo Model of Chemically Induced Hepatocellular Carcinoma," Japanese Journal of Cancer Research, Vol. 93, No. 10, 2002, pp. 1175-1181. doi:10.1111/j.1349-7006.2002.tb01220.x

[30] N. Murashige, M. Kami and M. Ikeda, “Aspirin, COX-2, and the Risk of Colorectal Cancer," The New England Journal of Medicine, Vol. 357, 2007, pp. 824-825; Author Reply pp. 824-825. doi:10.1056/NEJMc071797

[31] Y. J. Zhang, Y. J. Bao, Q. Dai, W. Y. Yang, P. Cheng, L. M. Zhu, B. J. Wang and F. H. Jiang, “mTOR Signaling Is Involved in Indomethacin and Nimesulide Suppression of Colorectal Cancer Cell Growth via a COX-2 Independent Pathway,” Annals of Surgical Oncology, Vol. 18, No. 2, 2011, pp. 580-588. doi:10.1245/s10434-010-1268-9

[32] M. Tsujii, S. Kawano and R. N. DuBois, "Cyclooxygenase-2 Expression in Human Colon Cancer Cells Increases Metastatic Potential," Proceedings of the National Academy of Sciences of the United States, Vol. 94, No. 7, 1997, pp. 3336-3340. doi:10.1073/pnas.94.7.3336

[33] M. Farooqui, Y. Li, T. Rogers, T. Poonawala, R. J. Griffin, C. W. Song and K. Gupta, "COX-2 Inhibitor Celecoxib Prevents Chronic Morphine-Induced Promotion of Angiogenesis, Tumour Growth, Metastasis and Mortality, 
without Compromising Analgesia,” British Journal of Cancer, Vol. 97, No. 11, 2007, pp. 1523-1531. doi:10.1038/sj.bjc.6604057

[34] B. Singh, J. A. Berry, A. Shoher, G. D. Ayers, C. Wei and A. Lucci, "COX-2 Involvement in Breast Cancer Metastasis to Bone,” Oncogene, Vol. 26, No. 26, 2007, pp. 37893796. doi:10.1038/sj.onc.1210154

[35] Q. Chen and J. Massague, "Molecular Pathways: Vcam-1 as a Potential Therapeutic Target in Metastasis," Clinical Cancer Research, Vol. 18, No. 20, 2012, pp. 5520-5525. doi:10.1158/1078-0432.CCR-11-2904

[36] J. P. Johnson, B. G. Stade, B. Holzmann, W. Schwable and G. Riethmuller, "De Novo Expression of Intercellular-Adhesion Molecule 1 in Melanoma Correlates with Increased Risk of Metastasis," Proceedings of the $\mathrm{Na}$ tional Academy of Sciences of the United States A, Vol. 86, No. 2, 1989, pp. 641-644. doi:10.1073/pnas.86.2.641

[37] C. Lawson, M. Ainsworth, M. Yacoub and M. Rose, "Ligation of ICAM-1 on Endothelial Cells Leads to Expression of VCAM-1 via a Nuclear Factor-KappaB-Independent Mechanism,” The Journal of Immunology, Vol. 162, No. 5, 1999, pp. 2990-2996.

[38] D. Zapolska-Downar and M. Naruszewicz, "Propionate Reduces the Cytokine-Induced VCAM-1 and ICAM-1 Expression by Inhibiting Nuclear Factor-Kappa B (NFKappaB) Activation," Journal of physiology and pharmacology, Vol. 60, No. 2, 2009, pp. 123-131.

[39] E. Kopp and S. Ghosh, "Inhibition of NF-Kappa B by Sodium Salicylate and Aspirin,” Science, Vol. 265, No. 5174, 1994, pp. 956-959. doi:10.1126/science.8052854

[40] C. Weber, W. Erl, A. Pietsch and P. C. Weber, “Aspirin Inhibits Nuclear Factor-Kappa B Mobilization and Monocyte Adhesion in Stimulated Human Endothelial Cells,” Circulation, Vol. 91, No. 7, 1995, pp. 1914-1917. doi:10.1161/01.CIR.91.7.1914

[41] D. K. Biswas, C. M. Ahlers, B. J. Dezube and A. B. Pardee, "Pentoxifylline and Other Protein Kinase C Inhibitors Down-Regulate HIV-LTR NF-Kappa B Induced Gene Expression,” Molecular Medicine, Vol. 1, No. 1, 1994, pp. 31-43.

[42] M. Roederer, P. A. Raju, F. J. Staal and L. A. Herzenberg, "N-Acetylcysteine Inhibits Latent HIV Expression in Chronically Infected Cells," AIDS Research and Human Retroviruses, Vol. 7, No. 6, 1991, pp. 563-567. doi:10.1089/aid.1991.7.563

[43] V. P. Sant, M. S. Nagarsenker, S. G. Rao and R. P. Gude, "Enhancement of Anti-Metastatic Activity of Pentoxifylline by Encapsulation in Conventional Liposomes and Sterically Stabilized Liposomes in Murine Experimental B16F10 Melanoma Model,” Journal of Pharmacy and Pharmacology, Vol. 52, No. 12, 2000, pp. 1461-1466. doi:10.1211/0022357001777667

[44] P. Redondo, E. Bandres, T. Solano, I. Okroujnov and J. Garcia-Foncillas, "Vascular Endothelial Growth Factor (VEGF) and Melanoma. N-Acetylcysteine Downregulates VEGF Production in Vitro," Cytokine, Vol. 12, No. 4, 2000, pp. 374-378. doi:10.1006/cyto.1999.0566
[45] T. Cai, G. Fassina, M. Morini, M. G. Aluigi, L. Masiello, G. Fontanini, F. D’Agostini, S. De Flora, D. M. Noonan and A. Albini, "N-Acetylcysteine Inhibits Endothelial Cell Invasion and Angiogenesis," Laboratory Investigation, Vol. 79, No. 9, 1999, pp. 1151-1159.

[46] S. Kawakami, Y. Kageyama, Y. Fujii, K. Kihara and H. Oshima, "Inhibitory Effect of N-Acetylcysteine on Invasion and MMP-9 Production of T24 Human Bladder Cancer Cells," Anticancer Research, Vol. 21, No. 1A, 2001, pp. 213-219.

[47] M. K. Jones, H. Wang, B. M. Peskar, E. Levin, R. M. Itani, I. J. Sarfeh and A. S. Tarnawski, "Inhibition of Angiogenesis by Nonsteroidal Anti-Inflammatory Drugs: Insight into Mechanisms and Implications for Cancer Growth and Ulcer Healing," Nature Medicine, Vol. 5, No. 12, 1999, pp. 1418-1423. doi:10.1038/70995

[48] S. Abiru, K. Nakao, T. Ichikawa, K. Migita, M. Shigeno, M. Sakamoto, H. Ishikawa, K. Hamasaki, K. Nakata and K. Eguchi, "Aspirin and NS-398 Inhibit Hepatocyte Growth Factor-Induced Invasiveness of Human Hepatoma Cells,” Hepatology, Vol. 35, No. 5, 2002, pp. 1117 1124. doi:10.1053/jhep.2002.32676

[49] M. Futakuchi, K. Ogawa, S. Tamano, S. Takahashi and T. Shirai, "Suppression of Metastasis by Nuclear Factor Kappab Inhibitors in an in Vivo Lung Metastasis Model of Chemically Induced Hepatocellular Carcinoma," Cancer Science, Vol. 95, No. 1, 2004, pp. 18-24. doi:10.1111/j.1349-7006.2004.tb03165.x

[50] V. Blank, P. Kourilsky and A. Israel, "NF-Kappa B and Related Proteins: Rel/Dorsal Homologies Meet AnkyrinLike Repeats,” Trends in Biochemical Sciences, Vol. 17, No. 4, 1992, pp. 135-140. doi:10.1016/0968-0004(92)90321-Y

[51] G. P. Nolan and D. Baltimore, "The Inhibitory Ankyrin and Activator Rel Proteins," Current Opinion in Genetics \& Development, Vol. 2, No. 2, 1992, pp. 211-220. doi:10.1016/S0959-437X(05)80276-X

[52] G. Dong, Z. Chen, T. Kato and C. Van Waes, "The Host Environment Promotes the Constitutive Activation of Nuclear Factor-KappaB and Proinflammatory Cytokine Expression during Metastatic Tumor Progression of Murine Squamous Cell Carcinoma," Cancer Research, Vol. 59, 14, 1999, pp. 3495-3504.

[53] L. Lin, G. N. DeMartino and W. C. Greene, "Cotranslational Biogenesis of NF-KappaB p50 by the 26S Proteasome,” Cell, Vol. 92, No. 6, 1998, pp. 819-828. doi:10.1016/S0092-8674(00)81409-9

[54] S. Huang, C. A. Pettaway, H. Uehara, C. D. Bucana and I. J. Fidler, "Blockade of NF-KappaB Activity in Human Prostate Cancer Cells Is Associated with Suppression of Angiogenesis, Invasion, and Metastasis," Oncogene, Vol. 20, No. 31, 2001, pp. 4188-4197. doi:10.1038/sj.onc.1204535

[55] H. M. Ko, K. H. Seo, S. J. Han, K. Y. Ahn, I. H. Choi, G. Y. Koh, H. K. Lee, M. S. Ra and S. Y. Im, "Nuclear Factor KappaB Dependency of Platelet-Activating FactorInduced Angiogenesis," Cancer Research, Vol. 62, No. 6, 2002, pp. 1809-1814. 